\title{
Latrunculin alters the actin-monomer subunit interface to prevent polymerization
}

\author{
Walter M. Morton*, Kathryn R. Ayscough† and Paul J. McLaughlin* \\ *The Wellcome Trust Centre for Cell Biology, Institute of Cell and Molecular Biology, University of Edinburgh, Swann Building, King's Buildings, Mayfield Road, \\ Edinburgh EH9 3JR, UK \\ †Institute of Biomedical and Life Sciences, Davidson Building, University of Glasgow, Glasgow G12 8QQ, UK \\ ‡e-mail:Paul.McLaughlin@ed.ac.uk
}

atrunculin-A is a drug that is capable of rapidly, reversibly and specifically disrupting the actin cytoskeleton ${ }^{1}$. The efficacy of its action has made it a compound of choice in many cell-biology laboratories, supplanting the classic actin-depolymerizing drug cytochalasin-D. One reason for this is that the mode of action of latrunculin seems to be less complex than that of cytochalasin. Whereas the latter affects the kinetics of actin-filament polymerization at both the barbed and pointed ends, latrunculin-A seems to associate only with actin monomers, thereby preventing them from repolymerizing into filaments ${ }^{2}$. The association of latrunculin with monomeric, rather than filamentous, actin gave us the opportunity to further our understanding of this interaction by detailed structural analysis of actin monomers using crystallographic techniques. Here we show the first high-resolution structure of an actin-disrupting drug in association with actin and discuss how its interactions with actin, and the conformational changes that its binding causes, may explain its mode of action within the cell.

Latrunculin (Fig. 1a) is purified from Latrunculia magnificans, a Red Sea sponge that exudes a noxious, red fluid that kills fish within minutes ${ }^{3}$. Two related compounds, latrunculin-A and latrunculin$\mathrm{B}$, isolated from the fluid were shown to depolymerize actin structures both in vitro and in vivo ${ }^{1,4}$. The in vitro studies showed that latrunculin binds only to the actin monomer and that the kinetics of this interaction are consistent with the complex being unable to polymerize ${ }^{2}$. Unlike cytochalasin, latrunculin can disrupt the actin cytoskeleton in yeast cells. This has enabled genetic studies to be carried out that have facilitated the identification of point mutations in the actin gene that cause cells to become resistant to the effects of the $\operatorname{drug}^{5}$ (Fig. 1b). The mutations that give rise to latrunculin resistance were found to be clustered around a distinct site, close to the nucleotide-binding site, which indicated that they might identify a potential binding site for latrunculin. However, as this site is not close to recognized subunit contacts in the filament, or to known binding sites for other proteins that associate with actin, the mechanism by which latrunculin exerts its effects has remained unclear.

Actin has never been known to crystallize in the absence of a binding protein that keeps it in a monodispersed state. Of the three known examples of such binding proteins, profilin is inappropriate as it promotes nucleotide exchange, whereas deoxyribonuclease 1 binds to domains that have been implicated, in studies of yeast genetics, in latrunculin binding. In contrast, gelsolin domain 1 in complex with actin $^{6}$ leaves these domains free and also reduces nucleotide exchange, as does latrunculin. We therefore soaked latrunculin-A

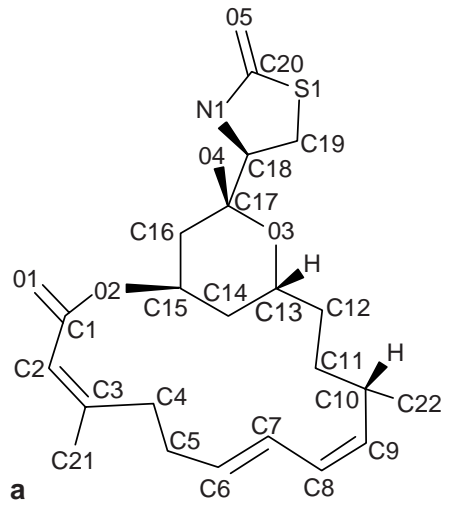

b

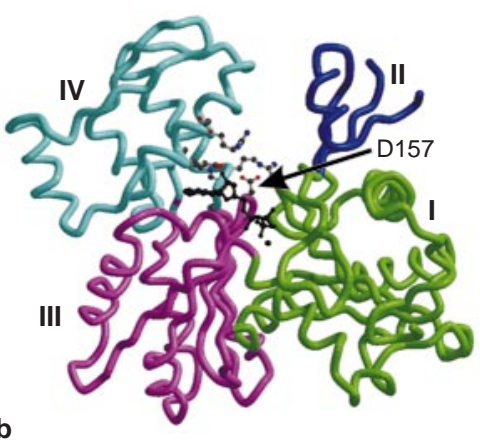

C

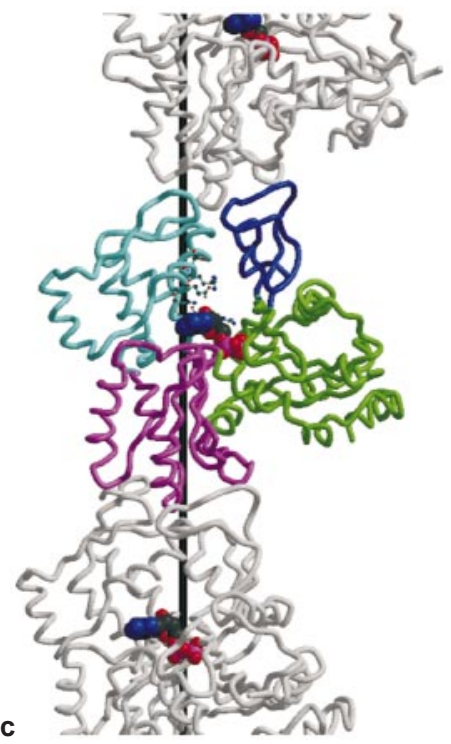

Figure 1 Latrunculin and actin structures. a, Chemical structure of latrunculin-A. b, Structure of actin. Actin domains in the crystal structure in complex with gelsolin ${ }^{6}$ are shown in green (I), blue (II), magenta (III) and cyan (IV). Bound nucleotide is shown as black balls and sticks; other balls and sticks represent corresponding residues from mutant yeast actins that are resistant to latrunculin. Diagrams were produced using Molscript ${ }^{13}$, Bobscript ${ }^{14}$ and Raster $3 D^{15}$. c, Holmes' fit of the actin crystal structure to an X-ray diagram of polymeric actin ${ }^{16}$. For clarity, only one strand is shown, concentrating on one subunit (coloured) and showing its relation to other subunits (grey) within the strand. 
a

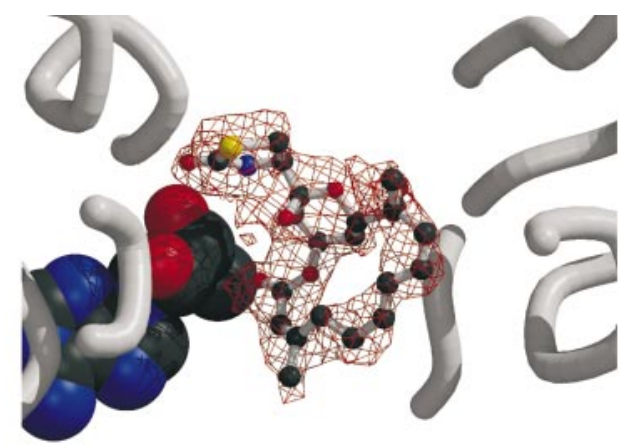

b
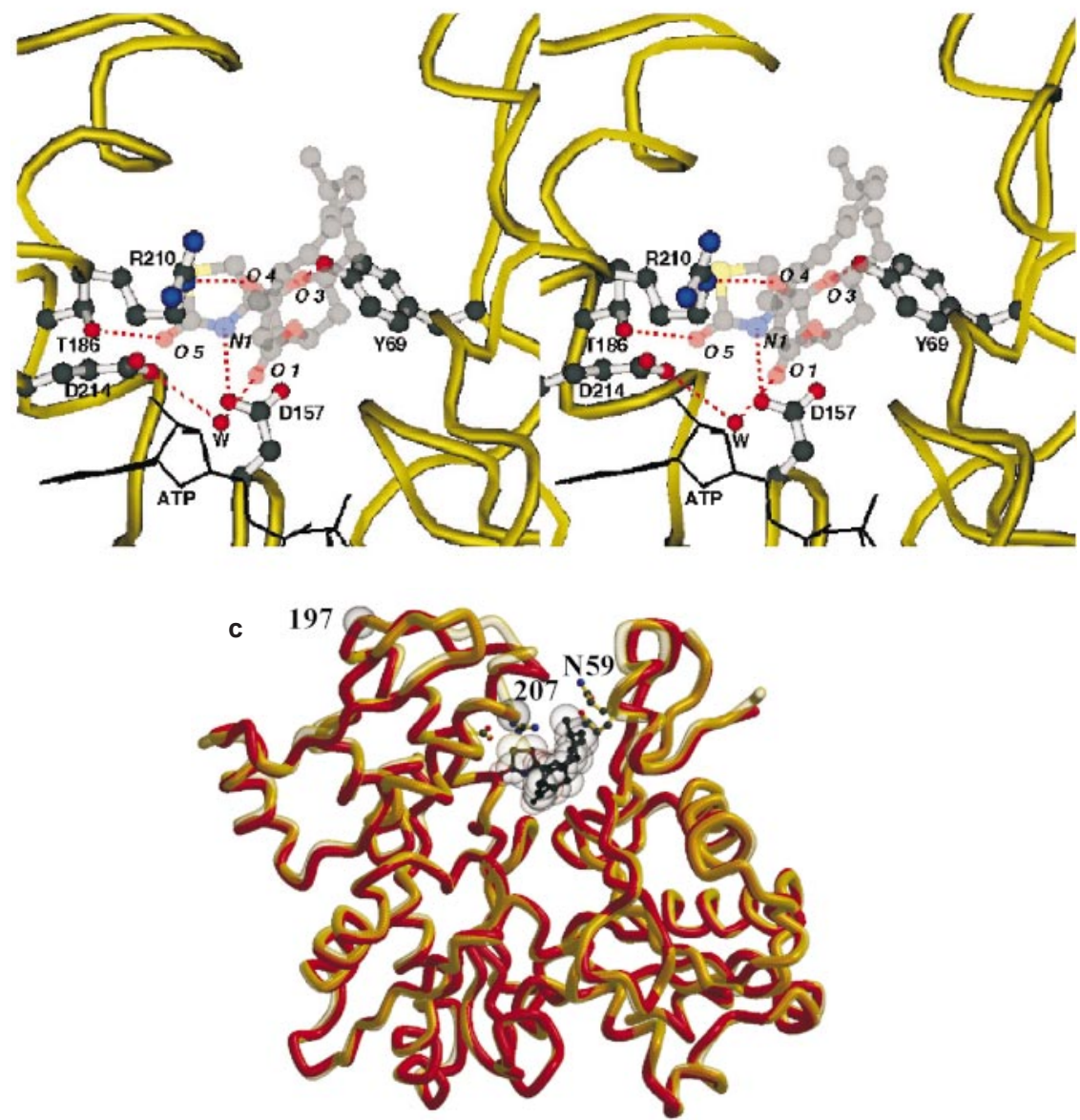

Figure 2 Crystal structure of the latrunculin-actin complex. a, Simulated annealing omit map showing electron density, at 2.5 r.m.s. deviation from the mean, for latrunculin A close to, but not in contact with, ATP (spacefilling). b. Stereodiagram showing hydrogen bonding between latrunculin $A$ and actin. The final model consisted of 4182 non-hydrogen atoms with r.m.s. deviation from ideal geometry of $0.019 \AA$ for bond distances and $2.4^{\circ}$ for bond angles, with $R_{\text {cryst }}=0.22$ and $R_{\text {free }}=0.28$ for all data. Latrunculin is shown as a semi-transparent ball-and-stick model, labelled in italics, with carbon atoms in grey. Every residue in actin contributing at least one hydrogen bond is shown, labelled with the residue name. ATP is shown as a wire-

into crystals of actin in complex with gelsolin domain 1. Difference mapping and subsequent crystallographic refinement of the model revealed the binding site (Fig. 2a), located above the actin nucleotidebinding site in a cleft between subdomains II and IV.

Specific contacts (such as hydrogen bonds) are formed at the Y69 site in subdomain II, at the T186 and R210 sites in subdomain IV and at the D157 site in subdomain III (Fig. 2b). The 2-thiazolid- frame skeleton for reference. W, water molecule bridging positions D214 in actin and 01 in latrunculin. The actin main-chain trace is shown in yellow. c, Comparison of the conformations of latrunculin-complexed (red) and uncomplexed (transparent) actin. Latrunculin is shown both as a black, ball-and-stick model and as a semi-transparent, space-filling model. Two conformations of the N59 site are shown, one clashing with the latrunculin model in the uncomplexed conformation and the other in its new position in the complex. Positions 197 and 207 are marked with transparent spheres. These delimit a large change in the loop between them. The salt bridge between the R206 and D187 sites is shown to the left of latrunculin.

inone group, which is rare in biology, fits neatly into a pocket and every polar latrunculin atom, except the $\mathrm{O} 2$-ester oxygen, forms a hydrogen bond with actin. These findings indicate that latrunculin may act to prevent dissociation of the nucleotide buried in the cleft by bridging subdomains II and IV.

The structure shows the validity of the genetic approach of mapping yeast mutants resistant to latrunculin onto the structure of 
actin. One mutation, D157E (ref. 7) is at a residue that forms a direct hydrogen bond with latrunculin. Although it is a conservative change, glutamate cannot be accommodated without steric clash. Other latrunculin-resistant mutants (Fig. 1b) map to the binding site ${ }^{5}$, although none of the sites at which substitution of a charged residue for alanine confers resistance bind directly to latrunculin. However, these mutations are likely to affect residues that do. Furthermore, none of these alanine mutations mapped to subdomain II, possibly because no charged residue in this domain is close to the binding site.

A further significant feature of the latrunculin-actin complex structure is the strong positive density around a salt bridge between the D187 and R206 sites. Although neither residue is directly involved in binding to latrunculin-A, R206, which is normally accessible at the subunit interface, is buried in the latruculin-actin complex. The importance of the R206 site has also been shown in yeast-actin alanine-scan studies, in which mutations of residues including R206 caused a dominant lethal phenotype in cells ${ }^{8}$. The D187-R206 salt bridge allows compensation of a charge that may otherwise inhibit latrunculin binding.

Changes in actin structure upon latrunculin binding are limited to specific regions, rather than affecting the entire structure (Fig. $2 c)$. In other actin structures, the two actin domains rearticulate, but here, the unliganded and liganded structures superpose well (0.34-Å r.m.s. deviation of main-chain atoms of core residues). The superposed structures (Fig. 2c) clearly show, however, that the N59 site in the unliganded structure occupies the same space as latrunculin does in the liganded structure. Upon latrunculin binding, the N59 site moves out of the way and there is a local rearrangement of the loop between the G55 and T66 sites in subdomain II. In addition, the loop between the G197 and E207 sites (subdomain IV) also moves (Fig. 2b), possibly as a result of the $2.4-\AA$ movement of the first carbon atom of R206 as its sidechain forms the salt bridge.

This first picture of an actin toxin bound in situ indicates that latrunculin may act by interfering with conformational changes that are necessary for polymerization. First, the structure shows that occupation of the latrunculin-binding site clamps ATP in its buried site by preventing movement between subdomains II and IV. Second, our structure shows that the two loops, G55 to T66 and G197 to E207, the movement of which accommodates latrunculin binding, propagate to the subunit-subunit interface at the tops of subdomains II and IV. In monomeric actin, these loops are among the most mobile, and dominant and partially dominant mutations are found within each, as shown by the alanine-scanning study ${ }^{5}$. Models of actin-filament structure predict that, upon incorporation into a filament, these loops become more ordered at the interface with the next subunit above. The structure shows that latrunculin binding to actin monomer exploits the very mobility of the loops, thereby influencing distant, critical subunit contacts, at a minimal penalty to its own binding at the better-ordered base of the cleft. Although it is conceivable that the gelsolin domain may prevent further conformational changes, we suggest that this is unlikely and that our structure provides a good model for the G-actin-latrunculin complex. Like latrunculin, gelsolin also limits nucleotide exchange, and in gelsolin-actin complex, domains I and III have the same disposition as in an actin-deoxyribonucleasel complex, in which these domains are unconstrained. We therefore propose that the toxin acts as a wedge that restricts the rotation of subdomains II and IV that is necessary for polymerization.

A further finding from the structural analysis is that the latrunculin-A hydrocarbon chain from C5 to C7 is exposed to solvent, thus forming few contacts with actin. Interestingly, latrunculin-B, but not Latrunculin-A, is found in sponges isolated near the Gulf of Eilat $^{1}$. Latrunculin-B only differs from latrunculin-A in that it lacks two carbons in the macrocyle ring that form one of its ethylene links. Our model is consistent with the idea that both compounds bind in similar ways. This idea is further supported by work on budding yeast, in which cells expressing mutant actins resistant to latrunculin-A have also been found to be resistant to latrunculin-B (K.R.A., unpublished observations). Thus, we now have clues as to where combinatorial chemistry may be applied to latrunculin to derive more specific actin-binding drugs.

An understanding of the mechanism of action for commonly used drugs is vital to facilitate interpretation of data in systems as complicated as a eukaryotic cell. Here we present the first high-resolution structure of actin in association with latrunculin and propose a mechanism of action on the basis of conformational changes induced by latrunculin binding.

\section{Methods}

Rabbit-muscle actin and recombinant human gelsolin domain 1 were purified, complexed and crystallized as described ${ }^{10}$. Gelsolin domain 1-actin crystals were soaked in a nominal 5-mM latrunculinA solution at $4^{\circ} \mathrm{C}$ for $4 \mathrm{~h}$ with mother liquor supplemented with $10 \%(v / v)$ DMSO, $20 \%(v / v)$ ethanol and $25 \%(v / v)$ glycerol. Data were obtained to $2.0 \AA$ at $100 \mathrm{~K}$ at beam line PX9.6 at the Daresbury synchrotron, UK. Coordinates that had been refined against native data collected at $100 \mathrm{~K}$ at DESY, Hamburg (beam line X11) were used as a starting model. The liganded structure was refined with REFMAC ${ }^{11}$ and SHELXL ${ }^{12}$.

RECEIVED 14 FEBRUARY 2000; REVISED 13 APRIL 2000; ACCEPTED 19 APRIL 2000; PUBLISHED 12 MAY 2000

1. Spector, I., Shochet, N. R., Kashman, Y. \& Groweiss, A. Science 219, 493-495 (1983).

2. Coué, M., Brenner, S. L., Spector, I. \& Korn, E. D. FEBS Lett. 213, 316-318 (1987).

3. Nèeman, I., Fishelson, L. \& Kashman, Y. Marine Biol. 30, 293-296 (1975).

4. Kashman, Y., Groweiss, A. \& Shmueli, U. Tetrahedron Lett. 21, 3629-3632 (1980).

5. Ayscough, K.R. et al. J. Cell Biol. 137, 399-416 (1997).

6. McLaughlin, P. J., Gooch, J. T., Mannherz, H-G. \& Weeds, A. G. Nature 364, 685-692 (1993).

7. Belmont, L. D., Patterson, G. M. L. \& Drubin, D. G. J.Cell Sci. 112, 1325-1336 (1999).

8. Wertman, K. F., Drubin, D. G. \& Botstein, D. Genetics 132, 337-350 (1992).

9. Page, R., Lindberg, U. \& Schutt, C. E. J. Mol. Biol. 280, 463-474 (1998).

10. Mannherz, H-G., Gooch, J., Way, M., Weeds, A. G. \& McLaughlin, P. J. J. Mol. Biol. 226, 899-901 (1992).

11. Murshudov, G. N., Vagin, A. A. \& Dodson, E. J. Acta Crystallogr. D 53, 240-255 (1997).

12. Sheldrick, G. M. \& Schneider, T. R. Methods Enzymol. 277, 319-343 (1997).

13. Kraulis, P. J. J. Appl. Crystallogr. 24, 946-950 (1991).

14. Esnouf, R. M. J. Mol. Graph. 15, 132-136 (1997).

15. Merritt, E. A. \& Bacon, D. J. Methods Enzymol. 277, 505-524 (1997).

16. Holmes, K. C., Popp, D., Gebhard, W. \& Kabsch, W. Nature 347, 44-49 (1990).

ACKNOWLEDGEMENTS

We are grateful for use of facilities at SRS, Daresbury, UK and at EMBL Hamburg (European Union HCMP LIP). This work was funded by the Wellcome Trust grant 040280 (to P.M.) and by Wellcome Career Development Award 050934 /Z/ 97 (to K.R.A.).

Correspondence and requests for materials should be addressed to P.M. Coordinates have been deposited in the protein data bank under accession numbers IESV for actin-gelsolin complex with latrunculin and IEQY for the complex without toxin. 